\title{
A national survey of antimicrobial prophylaxis in adult cardiac surgery across Canada
}

\author{
Fran L Paradiso-Hardy BScPhm MSc ${ }^{1,2}$, Patti Cornish BScPhm ${ }^{1}$, \\ Chantal Pharand PharmD ${ }^{3}$, Stephen E Fremes MD FRCSC ${ }^{4}$
}

\begin{abstract}
FL Paradiso-Hardy, P Cornish, C Pharand, SE Fremes. A national survey of antimicrobial prophylaxis in adult cardiac surgery across Canada. Can J Infect Dis 2002;13(1):21-27.
\end{abstract}

OBJECTIVE: To characterize national and regional patterns of antimicrobial prophylaxis in adult cardiac surgery across Canada. DESIGN: Retrospective, cross-sectional analysis.

SETTING: Thirty-three adult cardiac surgical centres across Canada.

INTERVENTIONS: A one-page questionnaire collecting information regarding institutional demographics and antimicrobial prophylaxis regimens for adult cardiac surgical procedures was mailed to all adult surgical centres across Canada. If a response was not received within one month, a second survey was mailed, followed by a telephone reminder within two weeks of the second mailing.

MAIN RESULTS: The overall response rate was 100\%. Prophylactic antimicrobials were used in all of the adult cardiac centres; single-agent prophylaxis was used in 97\% (32 of 33) of centres. Single-dose antimicrobial prophylaxis was used in only $3 \%$ (one of 33 ) of centres. Preoperative and postoperative antimicrobial prophylaxis regimens varied both between provinces and within provinces across Canada. Cefazolin was the antimicrobial used in $88 \%$ ( 38 of 43 ) and $87 \%$ (33 of 38) of the reported preoperative and postoperative prophylaxis regimens, respectively. Antimicrobial prophylaxis was initiated in the operating room $72 \%$ (26 of 36 ) of the time, and intraoperative supplemental antimicrobial doses were administered for cardiac procedures that took longer than a median of $4 \mathrm{~h}$ (range 4 to $8 \mathrm{~h}$ ). Overall, the median duration of antimicrobial prophylaxis treatment was $36 \mathrm{~h}$ (range 8 to $96 \mathrm{~h}$ ).
CONCLUSIONS: Despite the availability of various published guidelines, the present survey identified several areas for improvement with respect to antimicrobial prophylaxis in adult cardiac surgery across Canada.

Key Words: Antibiotics; Cardiac surgical procedures; Prophylaxis; Surgical wound infection

\section{Enquête nationale sur l'antibioprophylaxie en chirurgie cardiaque chez l'adulte au Canada}

OBJECTIF : Caractériser les habitudes d'antibioprophylaxie en chirurgie cardiaque chez l'adulte au Canada à l'échelle nationale et régionale. MODÈLE : Analyse transversale rétrospective.

CONTEXTE : Trente-trois centres de chirurgie cardiaque canadiens pour adultes.

INTERVENTION : Un questionnaire d'une page a été posté à tous les centres de chirurgie cardiaque pour adultes au Canada pour recueillir des données démographiques et des renseignements sur les schémas d'antibioprophylaxie utilisés lors d'interventions de chirurgie cardiaque chez l'adulte. Si aucune réponse n'était obtenue au bout d'un mois, un second questionnaire était expédié, suivi d'un rappel téléphonique dans les deux semaines suivant le second envoi postal.

PRINCIPAUX RÉSULTATS : Le taux de réponse global a été de 100 $\%$. L'antibioprophylaxie a été utilisée dans tous les centres de chirurgie cardiaque pour adultes. La mono-antibioprophylaxie a été utilisée dans 97 $\%$ des cas (32 centres sur 33); l'antibioprophylaxie à l'aide d'une seule dose a été utilisée dans $3 \%$ seulement des centres (1 sur 33).

Résumé à la page suivante

${ }^{1}$ Department of Pharmacy, ${ }^{2}$ Divisions of Cardiology and Clinical Pharmacology, Sunnybrook and Women's College Health Sciences Centre,

Toronto, Ontario; ${ }^{3}$ Department of Pharmacy, Hôpital Sacré-Coeur, Montreal, Quebec; 4Division of Cardiovascular Surgery,

Sunnybrook and Women's College Health Sciences Centre, Toronto, Ontario

Correspondence and reprints: Fran Paradiso-Hardy, Sunnybrook and Women's College Health Sciences Centre, 2075 Bayview Avenue,

Room E300, Toronto, Ontario M4N 3M5. Telephone 416-480-6755, fax 416-480-5887, e-mail fran.paradiso-hardy@swchsc.on.ca

Received for publication January 30, 2001. Accepted April 19, 2001 
L'antibioprophylaxie pré-opératoire et post-opératoire a varié entre les provinces et à l'intérieur des provinces. Au Canada, la céfazoline a été l'antibiotique utilisé dans $88 \%$ (38 sur 43) et dans $87 \%$ (33 sur 38) des antibioprophylaxies pré-opératoires et post-opératoires, respectivement. L'antibioprophylaxie a été amorcée en salle d'opération dans une proportion de $72 \%$ (26/36) et des doses d'antibiotiques peropératoires supplémentaires ont été administrées lorsque les interventions duraient plus longtemps que quatre heures en moyenne (de quatre à huit heures). Dans l'ensemble, la durée médiane de l'antibioprophylaxie a été de 36 heures (de 8 à 96 heures).

CONCLUSION : Malgré l'accessibilité à des principes directeurs publiés, l'enquête actuelle a identifié plusieurs secteurs où il y a place à l'amélioration en ce qui a trait à l'antibioprophylaxie lors de la chirurgie cardiaque chez l'adulte au Canada.
$\mathrm{P}$ ostoperative infections complicating cardiac surgery are associated with serious complications (eg, mediastinitis, osteomyelitis, sternal wound infections and endocarditis) that result in significant morbidity, mortality and increased costs (1-3). Despite evidence that antimicrobial prophylaxis is beneficial in reducing these complications, the optimal prophylactic regimen in cardiac surgery has not been defined (4-8). According to the 1999 Guidelines for Coronary Artery Bypass Graft Surgery from the American College of Cardiology and the American Heart Association (7), "Many centres, including those with training programs in cardiothoracic surgery, are not consistent in delivering or teaching effective use of perioperative antibiotics."

Although antimicrobial prophylaxis practice patterns in cardiac surgery have been documented for other geographical regions, the antimicrobial prophylaxis practice in adult cardiac surgery across Canada has not been documented to date (9-13). This information, in conjunction with the available literature, would be extremely useful for the development and/or revision of local guidelines for antimicrobial prophylaxis in cardiac surgery.

The objective of the present nationwide survey was to characterize national and regional patterns of antimicrobial prophylaxis in adult cardiac surgery across Canada.

\section{DATA AND METHODS}

\section{Adult cardiac surgical centres}

Thirty-three adult cardiac surgical centres were identified from information provided by Sulzer Medica Canada Inc (Toronto, Ontario) and the Cardiac Care Network of Ontario (Toronto, Ontario).

\section{Questionnaire}

The survey consisted of a one-page questionnaire that was designed to collect the following information regarding institutional demographics and antimicrobial prophylaxis regimens for adult cardiac surgical procedures: size and type of hospital; number of surgeons and cardiac procedures per year; standard preoperative and postoperative antimicrobial prophylaxis regimens; alternate regimens for penicillinallergic patients and high risk patients; criteria for high risk; use of preprinted standard orders; initiation of antimicrobial prophylaxis; and the use of intraoperative antimicrobial doses.

In April 2000, a cover letter (addressed to the Head of Cardiovascular Surgery) and questionnaire were mailed to all 33 adult cardiac surgical centres across Canada; a French version was sent to the 11 centres in Quebec. If a response was not received within one month, a second survery was mailed in May 2000, followed by a telephone reminder within two weeks of the second mailing. This study was approved by the Ethics Research Board at Sunnybrook and Women's College Health Sciences Centre (Toronto, Ontario).

\section{Statistical analysis}

Descriptive analysis was completed using SPSS for Windows (Release 10.0.5, SPSS Inc, USA).

\section{RESULTS}

The overall survey response rate was $100 \%$. Not all centres responded to every question. Institutional demographics are summarized in Table 1. Prophylactic antimicrobials were used by all of the adult cardiac surgical centres. Singleagent prophylaxis was used in 97\% (32 of 33) of centres; combination prophylaxis (ampicillin and cloxacillin) was used in only one of the 33 centres. Single dose antimicrobial prophylaxis with cefazolin was used in only $3 \%$ (one of 33) of the centres.

Standard preoperative antimicrobial prophylaxis regimens Cefazolin was the antimicrobial used in $88 \%$ (38 of 43) of the reported preoperative prophylactic regimens; vancomycin was used in only 7\% (three of 43 ) of the regimens. The preoperative dosages of cefazolin varied widely both between provinces and within provinces across Canada (Table 2).

\section{Initiation of antimicrobial prophylaxis}

Antimicrobial prophylaxis was initiated in the operating room $72 \%$ (26 of 36 ) of the time. In four centres (12\%), antimicrobial prophylaxis could be initiated in more than one location (Table 2).

Standard postoperative antimicrobial prophylaxis regimens Cefazolin was used in 87\% (33 of 38) of the various postoperative prophylaxis regimens. The postoperative dose regimens for cefazolin varied widely both between provinces and within provinces across Canada (Table 3 ).

\section{Duration of antimicrobial prophylaxis}

Overall, the median duration of antimicrobial prophylaxis was $36 \mathrm{~h}$ (range 8 to $96 \mathrm{~h}$ ). Prophylactic antimicrobials, whether used as a single agent or in combination, were stopped within 24 and $48 \mathrm{~h}$ of surgery $43 \%$ and $82 \%$ of the time, respectively. Regional differences in the duration of antimicrobial prophylaxis are summarized in Figure 1. 
TABLE 1

Demographics of Canadian cardiovascular surgical centres

\begin{tabular}{|c|c|c|c|c|c|c|c|c|c|c|}
\hline & $\begin{array}{l}\text { Overall } \\
\text { results }\end{array}$ & $\begin{array}{c}\text { British } \\
\text { Columbia }\end{array}$ & Alberta & Sask & Manitoba & Ontario & Quebec & NB & $\begin{array}{l}\text { Nova } \\
\text { Scotia }\end{array}$ & Nfld \\
\hline $\begin{array}{l}\text { Cardiovascular } \\
\text { surgical centres } \\
\text { per province (n) }\end{array}$ & 33 & 4 & 2 & 2 & 2 & 9 & 11 & 1 & 1 & 1 \\
\hline $\begin{array}{l}\text { Inpatient beds } \\
\text { per institution* }\end{array}$ & $558 \pm 243$ & $575 \pm 96$ & $800 \pm 283$ & $471 \pm 112$ & $705 \pm 417$ & $545 \pm 334$ & $500 \pm 182$ & 596 & 800 & 300 \\
\hline Type of institution & & & & & & & & & & \\
\hline $\begin{array}{l}\text { Teaching } \\
\text { Community }\end{array}$ & $\begin{array}{r}28 \\
5\end{array}$ & $\begin{array}{l}2 \\
2\end{array}$ & $\begin{array}{l}2 \\
0\end{array}$ & $\begin{array}{l}2 \\
0\end{array}$ & $\begin{array}{l}2 \\
0\end{array}$ & $\begin{array}{l}8 \\
1\end{array}$ & $\begin{array}{r}10 \\
1\end{array}$ & $\begin{array}{l}0 \\
1\end{array}$ & $\begin{array}{l}1 \\
0\end{array}$ & $\begin{array}{l}1 \\
0\end{array}$ \\
\hline Procedures per ye & & & & & & & & & & \\
\hline Total & $886 \pm 519$ & $838 \pm 275$ & $1111 \pm 127$ & $630 \pm 29$ & $555 \pm 50$ & $1244 \pm 630$ & $701 \pm 541$ & 700 & 1200 & 500 \\
\hline CABG & $645 \pm 372$ & $575 \pm 132$ & $792 \pm 153$ & $389 \pm 158$ & $423 \pm 39$ & $901 \pm 456$ & $565 \pm 411$ & 500 & NA & 400 \\
\hline $\begin{array}{l}\text { Valve with or } \\
\text { without } C A B G\end{array}$ & $203 \pm 127$ & $238 \pm 144$ & $275 \pm 35$ & $114 \pm 52$ & $115 \pm 15$ & $252 \pm 124$ & $183 \pm 157$ & 175 & NA & 100 \\
\hline Other & $44 \pm 81$ & $25 \pm 50$ & $44 \pm 62$ & $128 \pm 180$ & $18 \pm 4$ & $72 \pm 118$ & $21 \pm 41$ & 25 & NA & NA \\
\hline $\begin{array}{l}\text { Cardiac surgeons } \\
\text { per institution* }\end{array}$ & $5 \pm 2$ & $4 \pm 1$ & $6 \pm 1$ & $4 \pm 1$ & $4 \pm 1$ & $6 \pm 3$ & $3 \pm 2$ & 3 & 7 & 3 \\
\hline $\begin{array}{l}\text { Preprinted } \\
\text { order forms }\end{array}$ & $\begin{array}{c}71 \% \\
(22 / 31)^{\dagger}\end{array}$ & $\begin{array}{l}75 \% \\
(3 / 4)\end{array}$ & $\begin{array}{l}100 \% \\
(2 / 2)\end{array}$ & $\begin{array}{c}100 \% \\
(2 / 2)\end{array}$ & $\begin{array}{l}50 \% \\
(1 / 2)\end{array}$ & $\begin{array}{l}78 \% \\
(7 / 9)\end{array}$ & $\begin{array}{l}55 \% \\
(5 / 9)\end{array}$ & $\begin{array}{c}100 \% \\
(1 / 1)\end{array}$ & $\begin{array}{c}100 \% \\
(1 / 1)\end{array}$ & $\begin{array}{l}100 \% \\
(1 / 1)\end{array}$ \\
\hline
\end{tabular}

*Mean \pm SD; †Information not reported on two questionnaires. CABG Coronary artery bypass grafting; NA Not available; NB New Brunswick; Nfld Newfoundland; Sask Saskatchewan

TABLE 2

Preoperative antimicrobial prophylaxis regimens

\begin{tabular}{|c|c|c|c|c|c|c|c|c|c|c|}
\hline & $\begin{array}{l}\text { Overall } \\
\text { results }\end{array}$ & $\begin{array}{l}\text { British } \\
\text { Columbia }\end{array}$ & Alberta & Sask & Manitoba & Ontario & Quebec & NB & $\begin{array}{l}\text { Nova } \\
\text { Scotia }\end{array}$ & Nfld \\
\hline $\begin{array}{l}\text { Preoperative } \\
\text { prophylaxis }\end{array}$ & $\begin{array}{c}100 \% \\
(33 / 33)\end{array}$ & $\begin{array}{c}100 \% \\
(4 / 4)\end{array}$ & $\begin{array}{l}100 \% \\
(2 / 2)\end{array}$ & $\begin{array}{l}100 \% \\
(2 / 2)\end{array}$ & $\begin{array}{l}100 \% \\
(2 / 2)\end{array}$ & $\begin{array}{l}100 \% \\
(9 / 9)\end{array}$ & $\begin{array}{c}100 \% \\
(11 / 11)\end{array}$ & $\begin{array}{c}100 \% \\
(1 / 1)\end{array}$ & $\begin{array}{c}100 \% \\
(1 / 1)\end{array}$ & $\begin{array}{l}100 \% \\
(1 / 1)\end{array}$ \\
\hline \multicolumn{11}{|l|}{ Regimen $(n)^{*}$} \\
\hline $\begin{array}{l}\text { Ampicillin } 1 \mathrm{~g} \text { an } \\
\text { cloxacillin } 1 \mathrm{~g}\end{array}$ & 1 & - & - & - & - & - & 1 & - & - & - \\
\hline \multicolumn{11}{|l|}{ Cefazolin } \\
\hline Total & 38 & 6 & 2 & 3 & 3 & 11 & 9 & 2 & 1 & 1 \\
\hline $1 \mathrm{~g}$ & 23 & 1 & 1 & $1^{\dagger}$ & 2 & $9^{\ddagger}$ & 7 & 19 & 1 & - \\
\hline $2 \mathrm{~g}$ & 12 & 2 & 1 & $2^{\dagger}$ & 1 & $2^{\ddagger}$ & $2^{\S}$ & 19 & - & 1 \\
\hline $3 \mathrm{~g}$ & 2 & 2 & - & - & - & - & - & - & - & - \\
\hline $4 \mathrm{~g}$ & 1 & 1 & - & - & - & - & - & - & - & - \\
\hline Cloxacillin $1 \mathrm{~g}$ & 1 & - & - & - & - & - & 1 & - & - & - \\
\hline Vancomycin $1 \mathrm{~g}$ & 3 & $1^{* *}$ & - & - & - & - & $2^{\star \star}$ & - & - & - \\
\hline \multicolumn{11}{|c|}{ Initiation of antimicrobial prophylaxis $(n)^{\dagger \dagger}$} \\
\hline On ward & 2 & - & - & - & 1 & 1 & - & - & - & - \\
\hline On-call to OR & 4 & - & - & - & 2 & 1 & - & 1 & - & - \\
\hline Holding room & 4 & 1 & - & - & - & 2 & 1 & - & - & - \\
\hline In the OR & 26 & 4 & 2 & $2^{\ddagger \ddagger}$ & - & $6 \S \S$ & 10 & - & 1 & 1 \\
\hline
\end{tabular}

${ }^{*}$ Some institutions reported more than one preoperative prophylaxis regimen; ${ }^{\dagger}$ Cefazolin $1 \mathrm{~g}$ for patients weighing less than $75 \mathrm{~kg}$, cefazolin $2 \mathrm{~g}$ for patients weighing $75 \mathrm{~kg}$ or greater; ${ }^{\ddagger}$ Cefazolin $1 \mathrm{~g}$ for body mass index less than 30 , cefazolin $2 \mathrm{~g}$ for body mass index 30 or greater; $\$$ High risk patients (not defined); ${ }^{1}$ Cefazolin $1 \mathrm{~g}$ for patients weighing less than $100 \mathrm{~kg}$, cefazolin $2 \mathrm{~g}$ for patients weighing $100 \mathrm{~kg}$ or greater; ${ }^{*}$ Patients undergoing valve replacements or receiving prosthetic implants, patients with endocarditis; ${ }^{+\dagger}$ Antimicrobial prophylaxis was initiated in more than one location in four centres; ${ }^{\ddagger \neq}$ Administered with induction; $\$ \S A d m i n i s t e r e d$ before skin incision. OR Operating room; NB New Brunswick; Nfld Newfoundland; Sask Saskatchewan

Supplemental intraoperative antimicrobial doses

Overall, intraoperative supplemental antimicrobial doses were administered for cardiac surgical procedures taking longer than a median of $4 \mathrm{~h}$ (range 4 to $8 \mathrm{~h}$ ). Intraoperative supplemental antimicrobial doses were administered $60 \%$ of the time for cardiac procedures lasting longer than $4 \mathrm{~h}$. Regional differences are summarized in Figure 2.

Antimicrobial prophylaxis in high risk patients

The following criteria for high risk patients were provided 
TABLE 3

Postoperative antimicrobial prophylaxis regimens

\begin{tabular}{|c|c|c|c|c|c|c|c|c|c|c|}
\hline & $\begin{array}{l}\text { Overall } \\
\text { results }\end{array}$ & $\begin{array}{c}\text { British } \\
\text { Columbia }\end{array}$ & Alberta & Sask & Manitoba & Ontario & Quebec & NB & $\begin{array}{l}\text { Nova } \\
\text { Scotia }\end{array}$ & Nfld \\
\hline $\begin{array}{c}\text { Postoperative } \\
\text { prophylaxis }\end{array}$ & $\begin{array}{c}97 \% \\
(32 / 33)\end{array}$ & $\begin{array}{c}100 \% \\
(4 / 4)\end{array}$ & $\begin{array}{c}100 \% \\
(2 / 2)\end{array}$ & $\begin{array}{c}100 \% \\
(2 / 2)\end{array}$ & $\begin{array}{c}100 \% \\
(2 / 2)\end{array}$ & $\begin{array}{c}100 \% \\
(9 / 9)\end{array}$ & $\begin{array}{c}100 \% \\
(11 / 11)\end{array}$ & $\begin{array}{c}100 \% \\
(1 / 1)\end{array}$ & $\begin{array}{c}100 \% \\
(1 / 1)\end{array}$ & $\begin{array}{c}0 \% \\
(0 / 1)\end{array}$ \\
\hline \multicolumn{11}{|l|}{ Regimen $(n)^{\star}$} \\
\hline Ampicillin $1 \mathrm{~g}$ every $6 \mathrm{~h}$ & 1 & - & - & - & - & - & 1 & - & - & - \\
\hline \multicolumn{11}{|l|}{ Cefazolin } \\
\hline Total & 33 & 4 & 2 & 2 & 3 & 10 & 9 & 2 & 1 & - \\
\hline $0.5 \mathrm{~g}$ every $6 \mathrm{~h}$ & 1 & - & - & - & - & 1 & - & - & - & - \\
\hline $1.0 \mathrm{~g}$ every $6 \mathrm{~h}$ & 1 & - & - & - & - & - & 1 & - & - & - \\
\hline $1.0 \mathrm{~g}$ every $8 \mathrm{~h}$ & 23 & 2 & 1 & $2^{\dagger}$ & 2 & $8^{\ddagger}$ & 6 & $1 \S$ & 1 & - \\
\hline $2.0 \mathrm{~g}$ every $8 \mathrm{~h}$ & 6 & - & 1 & - & 1 & $1^{\ddagger}$ & 2 & $1 \S$ & - & - \\
\hline $2.0 \mathrm{~g}$ every $12 \mathrm{~h}$ & 1 & 1 & - & - & - & - & - & - & - & - \\
\hline $3.0 \mathrm{~g}$ every $6 \mathrm{~h}$ & 1 & 1 & - & - & - & - & - & - & - & - \\
\hline \multicolumn{11}{|l|}{ Cloxacillin } \\
\hline Total & 2 & - & - & - & - & - & 2 & - & - & - \\
\hline $1.0 \mathrm{~g}$ every $4 \mathrm{~h}$ & 1 & - & - & - & - & - & 1 & - & - & - \\
\hline $1.0 \mathrm{~g}$ every $6 \mathrm{~h}$ & 1 & - & - & - & - & - & 1 & - & - & - \\
\hline \multicolumn{11}{|l|}{ Vancomycin } \\
\hline Total & 2 & - & - & - & - & - & 2 & - & - & - \\
\hline $0.5 \mathrm{~g}$ every $8 \mathrm{~h}$ & 1 & - & - & - & - & - & & - & - & - \\
\hline $1.0 \mathrm{~g}$ every $12 \mathrm{~h}$ & 1 & - & - & - & - & - & $1^{1}$ & - & - & - \\
\hline
\end{tabular}

*Some institutions reported more than one postoperative prophylaxis regimen; ${ }^{\dagger}$ Cefazolin $2 \mathrm{~g}$ administered once patient was off cardiopulmonary bypass; ${ }^{\ddagger}$ Cefazolin $1 \mathrm{~g}$ for body mass index less than 30, cefazolin $2 \mathrm{~g}$ for body mass index 30 or greater; ${ }^{\S}$ Cefazolin $1 \mathrm{~g}$ every $8 \mathrm{~h}$ for patients weighing less than $100 \mathrm{~kg}$, cefazolin $2 \mathrm{~g}$ every $8 \mathrm{~h}$ for patients weighing $100 \mathrm{~kg}$ or greater; "Patients with valve replacements

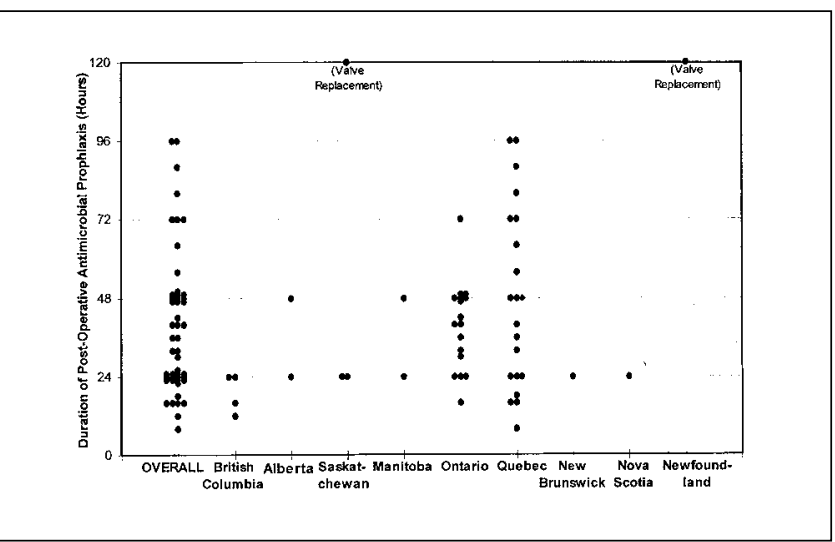

Figure 1) Duration of postoperative antimicrobial prophylaxis

by five centres (one in Nova Scotia, one in Quebec, two in Manitoba, one in Ontario): diabetes; older age (older than 75 years); endocarditis; sepsis; cardiogenic shock; steroid therapy; positive culture; and delayed sternal closure. Two centres $(6 \%)$ modified their antimicrobial prophylaxis regimen for high risk patients.

Antimicrobial prophylaxis regimens in valve surgery Two institutions (one in Vancouver, one in Quebec) used a different antimicrobial for prophylaxis in valve surgery (vancomycin) compared with coronary artery bypass surgery (cefazolin). The duration of antimicrobial prophylaxis was prolonged for valve replacement patients in two

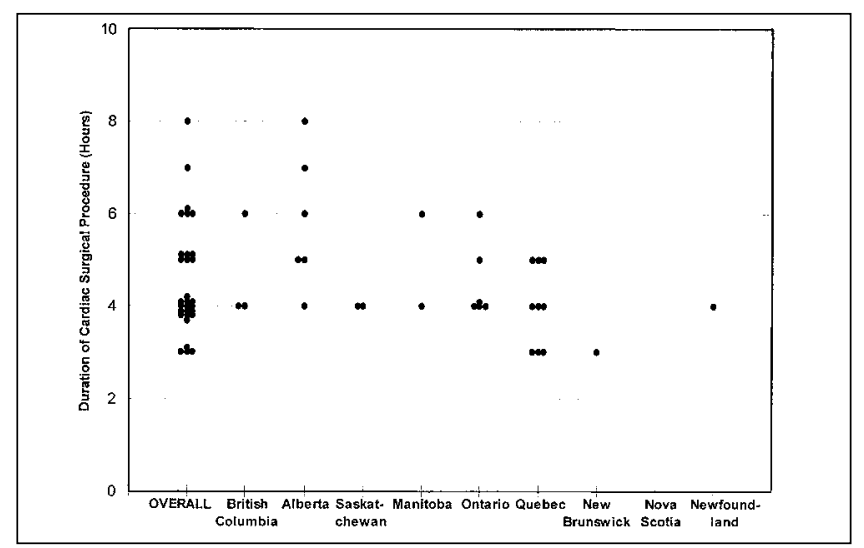

Figure 2) Duration of cardiac surgical procedure necessitating intraoperative antimicrobial supplement doses

centres (one in Saskatchewan, one in Newfoundland). Patients undergoing valve replacement at these centres received five days of prophylaxis versus single-dose (Saskatchewan) or 24-h (Newfoundland) for coronary artery bypass surgery.

Antimicrobial prophylaxis regimens in penicillin-allergic patients

Vancomycin was used 96\% (25 of 26) and 67\% (16 of 24) of the time for antimicrobial prophylaxis in penicillin-allergic patients preoperatively and postoperatively, respectively (Table 4). 
TABLE 4

Antimicrobial prophylaxis regimens in penicillin-allergic patients

\begin{tabular}{|c|c|c|c|c|c|c|c|c|c|c|}
\hline & $\begin{array}{l}\text { Overall } \\
\text { results } \\
(n=33)\end{array}$ & $\begin{array}{c}\text { British } \\
\text { Columbia } \\
(n=4)\end{array}$ & $\begin{array}{c}\text { Alberta } \\
(n=2)\end{array}$ & $\begin{array}{c}\text { Saskatchewan } \\
(n=2)\end{array}$ & $\begin{array}{c}\text { Manitob } \\
(n=2)\end{array}$ & $\begin{array}{c}\text { Ontario } \\
(n=9)\end{array}$ & $\begin{array}{c}\text { Quebec } \\
(n=11)\end{array}$ & $\begin{array}{c}\text { New } \\
\text { Brunswick } \\
(n=1)\end{array}$ & $\begin{array}{l}\text { Nova } \\
\text { Scotia } \\
(n=1)\end{array}$ & $\underset{(n=1)}{N \text { Nfld }}$ \\
\hline \multicolumn{11}{|l|}{ Preoperative regimen ( $n$ ) } \\
\hline Cefazolin $1 \mathrm{~g}$ & 1 & - & - & - & - & - & 1 & - & - & - \\
\hline \multicolumn{11}{|l|}{ Vancomycin } \\
\hline Total & 25 & 5 & 2 & 1 & - & 9 & 5 & 1 & 1 & 1 \\
\hline Dose NA & 11 & 1 & 1 & - & - & 6 & 2 & - & 1 & - \\
\hline $10-15 \mathrm{mg} / \mathrm{kg}$ & 1 & - & - & - & - & 1 & - & - & - & - \\
\hline $0.5 \mathrm{~g}$ & 1 & - & - & - & - & - & 1 & - & - & - \\
\hline $1.0 \mathrm{~g}$ & 11 & 3 & 1 & 1 & - & 2 & 2 & $1^{*}$ & - & 1 \\
\hline $1.5 \mathrm{~g}$ & 1 & 1 & - & - & - & - & - & - & - & - \\
\hline \multicolumn{11}{|l|}{ Postoperative regimen (n) } \\
\hline \multicolumn{11}{|l|}{ Cefazolin } \\
\hline Total & 2 & - & - & - & - & - & 2 & - & - & - \\
\hline $1 \mathrm{~g}$ every $6 \mathrm{~h}$ & 1 & - & - & - & - & - & 1 & - & - & - \\
\hline $1 \mathrm{~g}$ every $8 \mathrm{~h}$ & 1 & - & - & - & - & - & 1 & - & - & - \\
\hline \multicolumn{11}{|l|}{ Clindamycin } \\
\hline Total & 5 & - & - & 1 & - & 4 & - & - & - & - \\
\hline Dose NA & 2 & - & - & - & - & 2 & - & - & - & - \\
\hline $600 \mathrm{mg}$ every $8 \mathrm{~h}$ & 2 & - & - & 1 & - & $1(\mathrm{BMI}<30)$ & - & - & - & - \\
\hline $900 \mathrm{mg}$ every $8 \mathrm{~h}$ & 1 & - & - & - & - & $1(\mathrm{BMl} \geq 30)$ & - & - & - & - \\
\hline Erythromycin ${ }^{\dagger}$ & 1 & - & 1 & - & - & - & - & - & - & - \\
\hline \multicolumn{11}{|l|}{ Vancomycin } \\
\hline Total & 16 & 2 & 1 & 1 & - & 5 & 6 & 1 & - & - \\
\hline $10-15 \mathrm{mg} / \mathrm{kg}$ every $12 \mathrm{~h}$ & 1 & - & - & - & - & 1 & - & - & - & - \\
\hline $0.5 \mathrm{~g}$ every $8 \mathrm{~h}$ & 1 & - & - & - & - & - & 1 & - & - & - \\
\hline $0.5 \mathrm{~g}$ every $12 \mathrm{~h}$ & 4 & - & - & - & - & 2 & 2 & - & - & - \\
\hline $1.0 \mathrm{~g}$ every $8 \mathrm{~h}$ & 1 & - & - & - & - & 1 & - & - & - & - \\
\hline $1.0 \mathrm{~g}$ every $12 \mathrm{~h}$ & 9 & 2 & 1 & 1 & - & 1 & 3 & 1 & - & - \\
\hline
\end{tabular}

*Vancomycin $500 \mathrm{mg}$ administered once patient off cardiopulmonary bypass; ${ }^{\dagger}$ Dose not available. BMI Body mass index; NA Not available

\section{DISCUSSION}

Antimicrobial prophylaxis is generally recommended in the following situations: surgical procedures with high infection rates; surgeries involving implantation of prosthetic material; and surgeries in which the consequences of infection are particularly serious (4-8). Although cardiac surgery is generally considered a 'clean' surgery with an intrinsically low infection rate (ie, less than 5\%), postoperative infections complicating cardiac surgery are associated with potentially serious adverse outcomes $(1,2)$. Before the use of antimicrobial prophylaxis, postoperative infection rates ranged from $0.8 \%$ to $25 \%$, depending on the definition of wound infections and follow-up duration (14). A meta-analysis of 30 years of placebo controlled clinical trials demonstrated that antimicrobial prophylaxis for cardiac surgery (using penicillins or first-generation cephalosporins) reduces the risk of postoperative infections fivefold (odds ratio $4.96,95 \% \mathrm{CI}$ 2.06 to 9.72$)$ (14).

It is recommended that the selection of prophylactic antimicrobials be directed at the most likely infecting agents, Gram-positive bacteria (ie, Staphylococcus aureus and Staphylococcus epidermidis) (15). Despite the large num- ber of prospective, randomized, controlled clinical studies using various antimicrobials alone or in combination (including penicillins; first-, second- and third-generation cephalosporins; aminoglycosides; and vancomycin), no single regimen has emerged as clearly superior in preventing postoperative infections (14). First- or second-generation cephalosporins (cefazolin, cefuroxime) are among the most effective antimicrobials in reducing postoperative infections in cardiac surgery and are the agents of choice according to the 1999 American College of Cardiology/American Heart Association guidelines (7). Data supporting the benefit of extending the antibacterial spectrum with combination antimicrobial prophylaxis are limited (14). In a recent randomized, double-blind, comparative study in 1641 adults, no significant differences in postoperative infection rates were observed among those taking cefazolin (firstgeneration cephalosporin) versus cefuroxime or cefamandole (second-generation cephalosporins), 8.4\% versus 9.0\% or $8.6 \%$, respectively (16).

Studies have failed to demonstrate a clinically significant advantage of vancomycin over cephalosporins for routine antimicrobial prophylaxis (17-19). The emergence of 
TABLE 5

Regimens for antimicrobial prophylaxis in cardiac surgery

\begin{tabular}{|c|c|c|c|}
\hline Reference & Recommended antibiotic regimen & Alternative regimen* & Duration of prophylaxis \\
\hline $\begin{array}{l}\text { The Medical Letter on Drugs } \\
\text { and Therapeutics } 1999(4)\end{array}$ & Cefazolin or cefuroxime 1 to $2 \mathrm{~g}$ iv & Vancomycin $1 \mathrm{~g}$ iv & $\begin{array}{l}\text { Single preoperative dose and } \\
\text { one additional dose after } \\
\text { CPB (optional) }\end{array}$ \\
\hline $\begin{array}{l}\text { American Society of } \\
\text { Hospital Pharmacists (5) }\end{array}$ & $\begin{array}{l}\text { Cefazolin } 1 \mathrm{~g} \text { iv preoperatively and } \\
\text { every } 8 \mathrm{~h} \text { or cefuroxime } 1.5 \mathrm{~g} \text { iv } \\
\text { preoperatively and every } 12 \mathrm{~h}\end{array}$ & $\begin{array}{l}\text { Vancomycin } 1 \mathrm{~g} \text { iv } \\
\text { with or without gentamicin } \\
2 \mathrm{mg} / \mathrm{kg} \text { iv }\end{array}$ & Up to $72 \mathrm{~h}$ \\
\hline Waddell et al (6) & $\begin{array}{l}\text { Cefazolin } 1 \mathrm{~g} \text { iv or cefuroxime } 1.5 \mathrm{~g} \text { iv } \\
\text { preoperatively }\end{array}$ & No recommendation & $\begin{array}{l}\text { Optimal duration not clearly } \\
\text { defined - single dose may } \\
\text { not be sufficient }\end{array}$ \\
\hline Eagle et al (7) & $\begin{array}{l}\text { Cefuroxime } 1.5 \mathrm{~g} \text { iv preoperatively, } \\
1.5 \mathrm{~g} \text { iv after CPB and } 1.5 \mathrm{~g} \text { iv } \\
\text { every } 12 \mathrm{~h} \text {, or cefazolin } 1 \mathrm{~g} \text { iv } \\
\text { preoperatively, } 1 \mathrm{~g} \text { at sternotomy, } \\
1 \mathrm{~g} \text { after CPB and } 1 \mathrm{~g} \text { every } 6 \mathrm{~h}\end{array}$ & $\begin{array}{l}\text { Vancomycin } 1 \mathrm{~g} \text { iv } \\
\text { preoperatively and } 1 \mathrm{~g} \\
\text { iv every } 12 \mathrm{~h}\end{array}$ & 24 to $48 \mathrm{~h}$ \\
\hline Martin (8) & $\begin{array}{l}\text { Cefazolin } 2 \mathrm{~g} \text { iv preoperatively } \\
\text { and } 1 \mathrm{~g} \text { every } 8 \mathrm{~h} \text { or cefuroxime } 1.5 \mathrm{~g} \\
\text { iv preoperatively and } 0.75 \mathrm{~g} \\
\text { iv every } 6 \mathrm{~h}\end{array}$ & $\begin{array}{l}\text { Vancomycin } 15 \mathrm{mg} / \mathrm{kg} \text { iv } \\
\text { preoperatively, } 7.5 \mathrm{mg} / \mathrm{kg} \\
\text { after CPB and } 10 \mathrm{mg} / \mathrm{kg} \text { iv } \\
\text { every } 8 \mathrm{~h}\end{array}$ & 24 to $48 \mathrm{~h}$ \\
\hline
\end{tabular}

${ }^{\star}$ For use in beta-lactam allergy or for hospitals in which methicillin-resistant Staphylcoccus aureus or Staphylococcus epidermidis are a frequent cause of post operative infection. CPB Cardiopulmonary bypass; iv Intravenously

vancomycin-resistant enterococci has restricted the use of vancomycin for antimicrobial prophylaxis to patients with a serious beta-lactam allergy or for hospitals in which methicillin-resistant $S$ aureus and/or $S$ epidermidis are a frequent cause of postoperative infections (20).

Our national survey demonstrated that, although the preoperative and postoperative antimicrobial prophylaxis dose regimens varied widely both between provinces and within provinces across Canada, the majority of centres used singleagent prophylaxis with the first-generation cephalosporin, cefazolin. The use of this agent may be attributed to its continued efficacy in preventing postoperative infections, favourable toxicity profile and reasonable cost.

Risk factors for postoperative infection include diabetes, obesity, smoking, prolonged preoperative hospital stay, chronic obstructive pulmonary disease and prolonged cardiopulmonary bypass $(14,21)$. Prophylactic antimicrobial prophylaxis does not appear to decrease postoperative infections associated with these risk factors, whereas other techniques (such as aggressive perioperative glucose control) have been shown to have a beneficial effect $(22,23)$. In our survey, $6 \%$ and $9 \%$ of centres modified their antimicrobial prophylaxis regimen for high risk patients and valve replacement patients, respectively.

It is established that for prophylaxis to be effective, the initial dose of the antimicrobial agent must be administered within 30 to $60 \mathrm{~min}$ of the time of incision to ensure a bactericidal concentration of the drug in serum and tissues $(21,24)$. For most procedures, scheduling administration at the time of induction of anesthesia before skin incision ensures adequate concentrations during the period of potential contamination (5). In our survey, antimicrobial prophylaxis was initiated either on the ward, on-call to the operating room (OR) or in the OR holding room $26 \%$ of the time. Optimal initiation of antimicrobial prophylaxis in the OR occurred $74 \%$ of the time.

It is also established that when an antimicrobial with a relatively short half-life (eg, cefazolin) is administered preoperatively, an additional intraoperative dose may be necessary to maintain bactericidal tissue concentrations during lengthy procedures (ie, those lasting longer than 3 to $4 \mathrm{~h}$ ) $(4,25,26)$. In our survey, intraoperative supplemental antimicrobial doses were only administered $60 \%$ of the time for cardiac procedures lasting longer than $4 \mathrm{~h}$.

The optimal duration of antimicrobial prophylaxis in cardiac surgery remains controversial. According to the most recent 1999 American College of Cardiology/ American Heart Association guidelines, a $24 \mathrm{~h}$ course of intravenous antimicrobial prophylaxis is as efficacious as prolonged regimens ( $48 \mathrm{~h}$ or longer), even in high risk patients (7). In our survey, antimicrobials were stopped within 24 h of surgery only $42 \%$ of the time.

Because prolonged antimicrobial prophylaxis use after cardiac surgery may result in increased drug toxicity, increased risk of acquired antibiotic resistance, alterations in a patient's gastrointestinal flora and increased cost, it should generally be avoided. Recently, an observational four-year cohort study demonstrated that prolonging antimicrobial prophylaxis beyond $48 \mathrm{~h}$ after cardiac surgery did not further decrease the risk of surgical site infections compared with shorter durations (shorter than $48 \mathrm{~h}$ ) of prophylaxis (27). In our survey, prolonged antimicrobial prophylaxis (48 h or longer) occurred 19\% of the time. There is no evidence to support continuing prophylaxis until removal of the chest tubes.

Table 5 provides a summary of recommended prophylactic regimens from various consensus references and highlights the diversity of opinion regarding postoperative 
antimicrobial prophylaxis (4-8). According to these various guidelines, the preoperative antibiotic regimen was appropriate in $21 \%$ to $88 \%$ of institutions included in this survey, the postoperative antibiotic regimen was appropriate in 3\% to $64 \%$ of institutions included in this survey, and the duration of antimicrobial prophylaxis was appropriate in $67 \%$ to 91\% of institutions included in this survey.

Antimicrobial prophylaxis practice patterns in cardiac surgery documented in the United Kingdom, the United States and Germany have demonstrated inconsistent and ineffective use of antimicrobial prophylaxis (9-13). Frequent errors included incorrect timing of initial dose, prolonged duration of prophylaxis, incorrect selection of antimicrobial and inappropriate dose regimens. To our knowledge, this is the first survey characterizing national and regional patterns of antimicrobial prophylaxis in adult cardiac surgery across Canada.

Randomized trials do not provide data on the effect of antimicrobial prophylaxis in actual clinical practice. A major limitation of our study was the inability to document patient outcomes to assess the effectiveness of the various

\section{REFERENCES}

1. Milano C, Kesler K, Archibald N, et al. Mediastinitis after coronary artery bypass graft surgery: risk factors and long-term survival. Circulation 1995;92:2245-51.

2. Nelson R, Dries D. The economic implications of infection in cardiac surgery. Ann Thorac Surg 1986;42:240-6.

3. Hall R, Ash A, Ghali W, et al. Hospital cost of complications associated with coronary artery bypass graft surgery. Am J Cardiol 1997;79:1680-2.

4. Antimicrobial prophylaxis in surgery. Med Lett Drug Ther 1999;41:75-9.

5. American Society of Health System Pharmacists. ASHP Therapeutic Guidelines on Antimicrobial Prophylaxis in Surgery.

Am J Health Syst Pharm 1999;56:1839-88.

6. Waddell TK, Rotstein OD, Canadian Infectious Disease Society. Antimicrobial prophylaxis in surgery. Committee on Antimicrobial Agents. Can Med Assoc J 1994;151:925-31.

7. Eagle K, Guyton R, Davidoff R, et al. ACC/AHA Guidelines for Coronary Artery Bypass Graft Surgery: A Report of the American College of Cardiology/American Heart Association Task Force on Practice Guidelines (Committee to Revise the 1991 Guidelines for Coronary Artery Bypass Graft Surgery). J Am Coll Cardiol 1999;34:1262-346.

8. Martin C. Antimicrobial prophylaxis in surgery: general concepts and clinical guidelines. French Study Group on Antimicrobial Prophylaxis in Surgery, French Society of Anesthesia and Intensive Care. Infect Control Hosp Epidemiol 1994;15:463-71.

9. Kappstein I, Daschner F. Use of perioperative antibiotic prophylaxis in selected surgical procedures - results of a survey in 889 surgical department in German hospitals. Infection 1991;19:391-4.

10. Parry G, Holden S, Shabbo F. Antibiotic prophylaxis for cardiac surgery: current United Kingdom practice. Br Heart J 1993;70:585-6.

11. Widdison A, Pope N, Brown E. Survey of guidelines for antimicrobial prophylaxis in surgery. J Hosp Infect 1993;25:199-205.

12. Woods M, Tillman D. Antibiotic prophylaxis in cardiothoracic surgery 1990; results of a third survey. Hosp Pharm 1992;27:406-7.

13. Slaughter M, Olson M, Lee J, Ward H. A fifteen-year wound surveillance study after coronary artery bypass. Ann Thorac Surg 1993;56:1063-8.

14. Kreter B, Woods M. Antibiotic prophylaxis for cardiothoracic operations; meta-analysis of thirty years of clinical trials. J Thorac Cardiovasc Surg 1992;104:590-9.

15. Ariano R, Zhanel G. Antimicrobial prophylaxis in coronary bypass prophylaxis regimens used in adult cardiac surgery across Canada.

\section{CONCLUSIONS}

Despite the availability of various published guidelines for antimicrobial prophylaxis in adult cardiac surgery, the present survey identified several areas for improvement in adult cardiac surgery across Canada. These include initiating antimicrobial prophylaxis in the OR, using intraoperative supplemental antimicrobial doses for cardiac procedures lasting longer than $4 \mathrm{~h}$ and shortening the duration of antimicrobial prophylaxis to 24 to $48 \mathrm{~h}$. The results from this survey have been provided to all cardiac centres across Canada, and may be used to develop and/or revise evidence-based institutional guidelines. A follow-up survey is planned in one year to determine whether this information improved practice patterns.

ACKNOWLEDGEMENTS: Gratitude is extended to Claudia Bucci for her invaluable input into the development of the questionnaire. surgery: A critical appraisal. Ann Phamacother 1991;25:478-84.

16. Townsend T, Reitz B, Bilker W, Bartlett J. Clinical trial of cefamandole, cefazolin, and cefuroxime for antibiotic prophylaxis in cardiac operations. J Thorac Cardiovasc Surg 1993;106:664-70.

17. Maki D, Bohn M, Stolz S, Kroncke G, Acher C, Myerowitz P. Comparative study of cefazolin, cefamandole, and vancomycin for surgical prophylaxis in cardiac and vascular operations. A doubleblind randomized trial. J Thorac Cardiovasc Surg 1992;104:1423-34.

18. Saginur R, Croteau D, Bergeron M, the ESPRIT Group. Comparative efficacy of teicoplanin and cefazolin for cardiac operation prophylaxis in 3027 patients. J Thorac Cardiovasc Surg 2000;120:1120-30.

19. Vuorisalo S, Pokela R, Syrjala H. Comparison of vancomycin and cefuroxime for infection prophylaxis in coronary artery bypass surgery. Infect Control Hosp Epidemiol 1998;19:234-9.

20. Recommendations for preventing the spread of vancomycin resistance: recommendations of the Hospital Infection Control Practice Advisory Committee (HICPAC). MMWR Morbid Mortal Wkly Rep 1994;44:1-13.

21. Mangram A, Horan T, Pearon M, Silver L, Jarvis W. Guidelines for prevention of surgical site infection. Infect Control Hosp Epidemiol 1999;20:250-78.

22. Niederhauser U, Vogt M, Vogt P, Genoni M, Kunzli A, Turina M. Cardiac surgery in a high-risk group of patients: is prolonged postoperative antibiotic prophylaxis effective? J Thorac Cardiovasc Surg 1997;114:162-8.

23. Furnary A, Grunkemeier G, Floten H, Swanson J, Gately H, Starr A. Continuous intravenous insulin infusion reduces the incidence of deep sternal wound infection in diabetic patients after cardiac surgical procedures. Ann Thorac Surg 1999;67:352-60.

24. Classen D, Evans R, Pestotnik S, Horn S, Menlove R, Burke J. The timing of prophylactic administration of antibiotics and the risk of surgical-wound infection. N Engl J Med 1992;326:281-6.

25. Dellinger E, Gross P, Barrett T, et al. Quality standard for antimicrobial prophylaxis in surgical procedures. Clin Infect Dis 1994;18:422-7.

26. Nagachinta T, Stephens M, Reitz B, Polk B. Risk factors for surgical wound infection following cardiac surgery. J Infect Dis 1987;156:967-73.

27. Harbarth S, Samore M, Lichtenberg D, Carmeli Y. Prolonged antibiotic prophylaxis after cardiovascular surgery and its effect on surgical site infections and antimicrobial resistance. Circulation 2000;101:2916-21. 


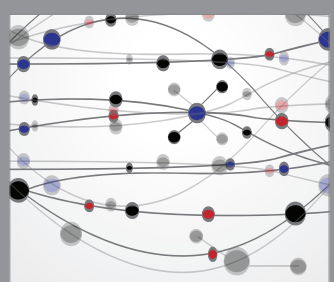

The Scientific World Journal
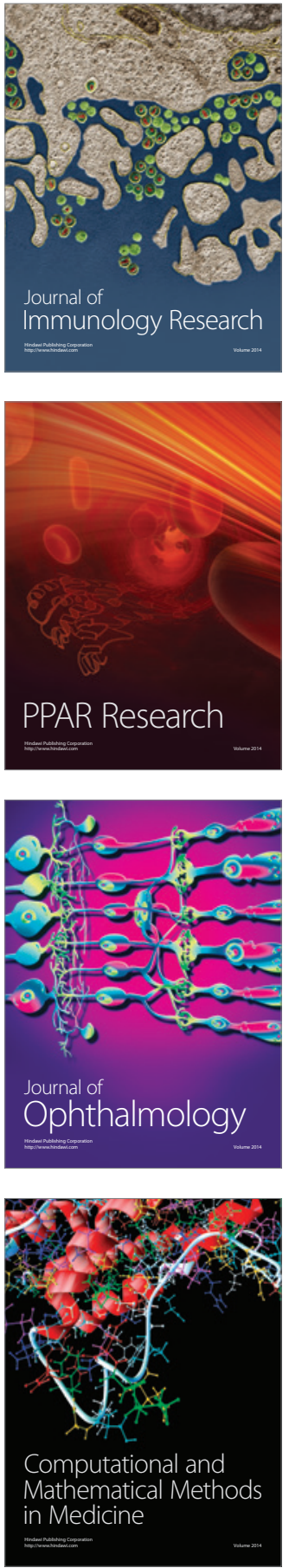

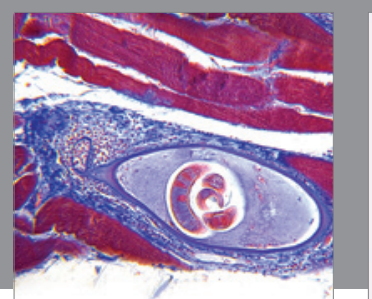

Gastroenterology Research and Practice

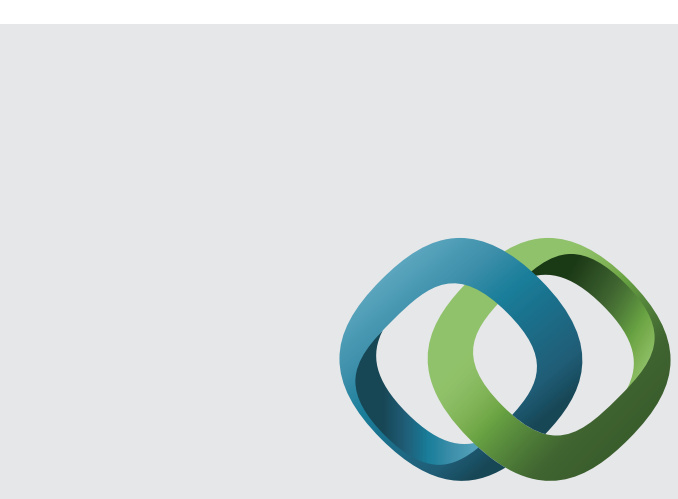

\section{Hindawi}

Submit your manuscripts at

http://www.hindawi.com
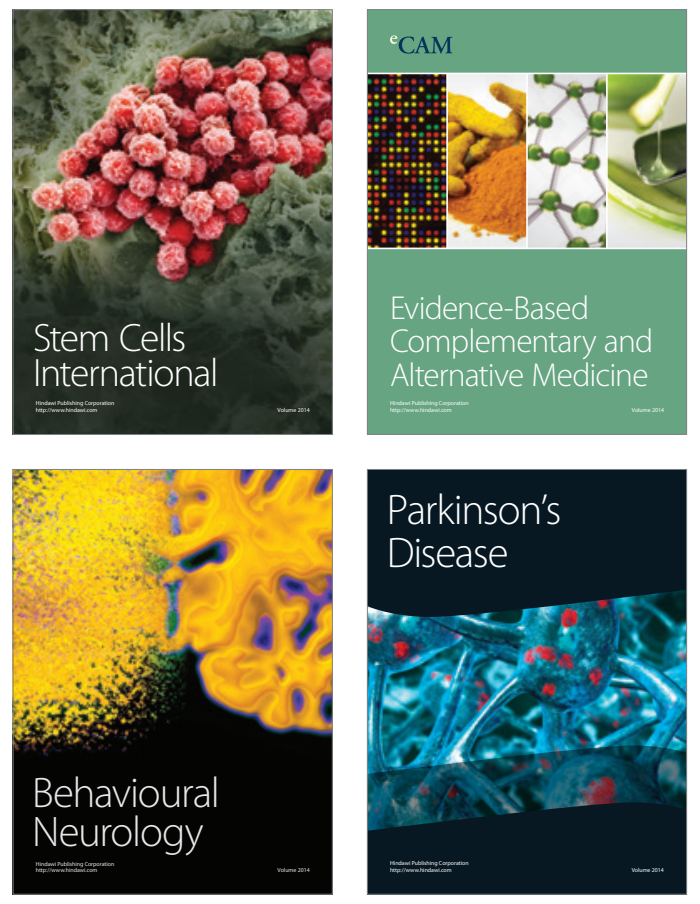
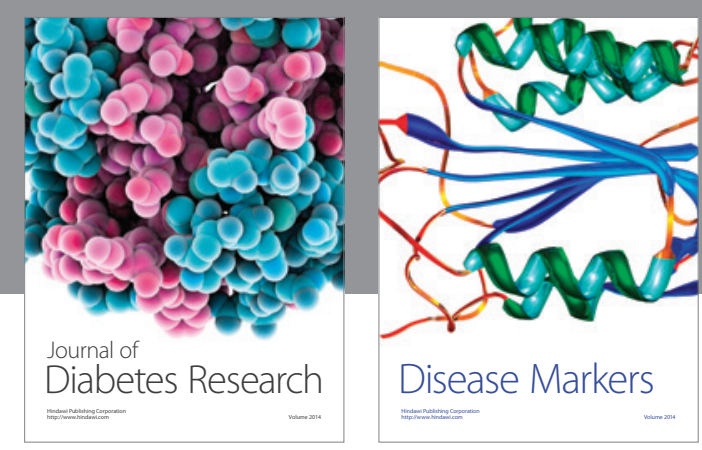

Disease Markers
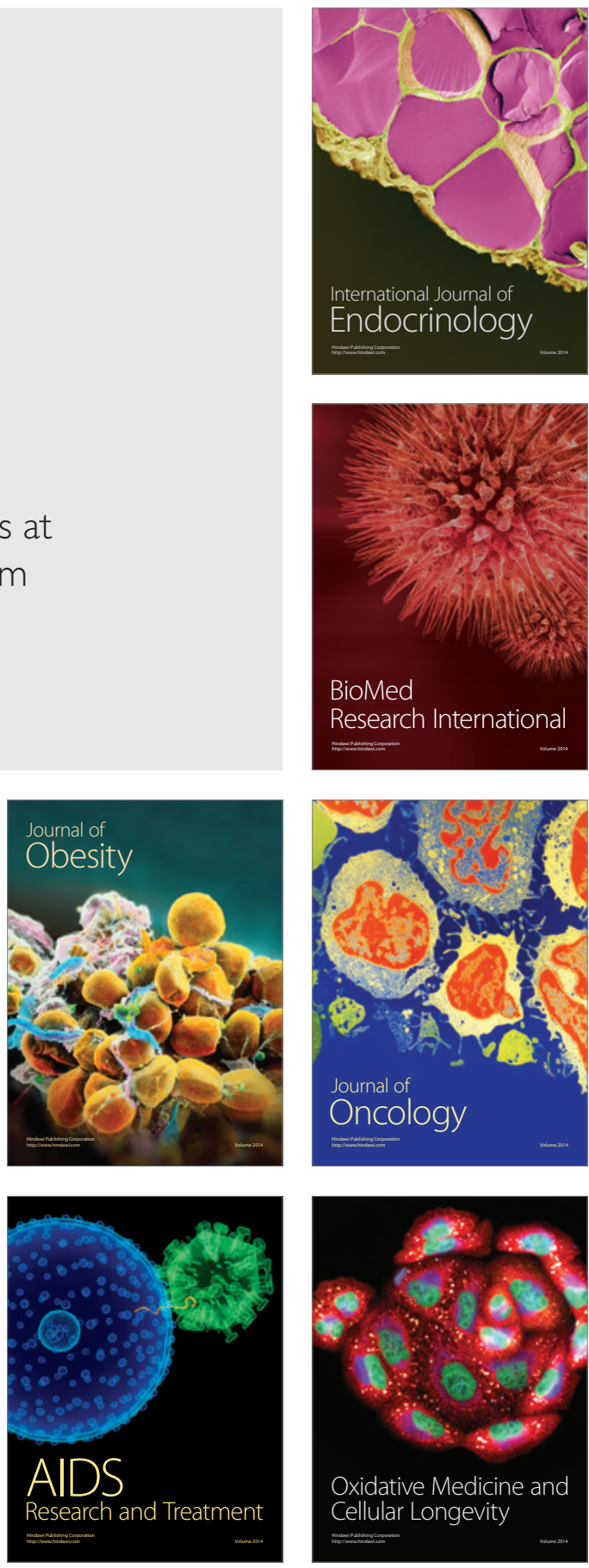